\title{
Very Low-Power Consumption Analog Pulse Processing ASIC for Semiconductor Radiation Detectors
}

\author{
K.O. Wessendorf', J.C. Lund', B.A. Brunett ${ }^{3}$, G.R. Laguna', and J.W. Clement \\ 'Sandia National Laboratories, P.O. Box 5800, Albuquerque, New Mexico 87185 \\ 'Sandia National Laboratories, P.O. Box 969, Livermore, California 94551 \\ ${ }^{3}$ Carnegie Mellon University, Pittsburgh, PA 15213
}

\begin{abstract}
We describe a very-low power consumption circuit for processing the pulses from a semiconductor radiation detector. The circuit was designed for use with a cadmium zinc telluride (CZT) detector for unattended monitoring of stored nuclear materials. The device is intended to be battery powered and operate at low duty-cycles over a long period of time. This system will provide adequate performance for medium resolution gamma-ray pulse-height spectroscopy applications. The circuit incorporates the functions of a charge sensitive preamplifier, shaping amplifier, and peak sample and hold circuit. An application specific integrated circuit (ASIC) version of the design has been designed, built and tested. With the exception of the input field effect transistor (FET), the circuit is constructed using bipolar components. In this paper the design philosophy and measured performance characteristics of the circuit are described.
\end{abstract}

\section{INTRODUCTION}

Conventional pulse processing electronics for radiation detectors consume relatively large amounts of power and are often unsuitable for use outside the laboratory. Lower power charge preamplifier circuits have been successfully developed $[1,2]$. However, for long term unattended monitoring applications, the entire pulse processing system [3,4] (preamplifier, shaping amplifier, sample-hold) must also consume very little power. We are interested in deploying circuits in a nuclear monitoring application in which long operating lifetime is critical. For the duty cycles that we envision in our application, this energy budget translates into an average power consumption of $14 \mathrm{~mW}$ for the pulse processing circuit.

\section{DESIGN INTENT}

The circuit we designed is intended to perform the same functions as a charge sensitive preamplifier, shaping amplifier, and a peak sample and hold circuit. However, to minimize packaging volume and power consumption, the three functions are integrated on one ASIC. The pulse processing ASIC is designed to interface to a flash analog to digital converter $(\mathrm{ADC})$ for subsequent pulse height analysis. Low average power was achieved by making the high-current functions enabled only when the desired input pulse amplitude level is detected. Novel circuit design techniques were used to minimize power consumption while providing good linearity and moderately low-noise circuit performance.
The circuit was designed to operate off of a $7 \mathrm{~V}( \pm 3.5 \mathrm{~V})$ power supply and used with a CZT detector to provide pulse height spectroscopy from 0 to $1.0 \mathrm{MeV}$ with an energy resolution of at least $20 \mathrm{keV}$ FWHM. Assuming a mean electron-hole pair creation energy in CZT of $5.0 \mathrm{eV}$ these design specification translate into a "charge gain" of $58.6 \mu \mathrm{V} / \mathrm{keV}(133 \mathrm{mV} / \mathrm{fC})$ and an equivalent noise charge (ENC) [5] of less than 1700 electrons rms. By altering the effective feedback capacitance in the circuit, it should be possible to alter the gain for use with other detectors besides CZT. For instance, by increasing the "charge gain" of the circuit it should be possible to use the circuit as a readout for silicon $\mathrm{p}-\mathrm{i}-\mathrm{n}$ detectors attached to scintillators.

\section{OVERVIEW OF ASIC DESIGN}

The ASIC performs the three analog pulse processing functions used in a semiconductor pulse height spectrometer: charge amplification, shaping, and peak sensing. A simplified block diagram of the ASIC circuit is shown in Figure 1.

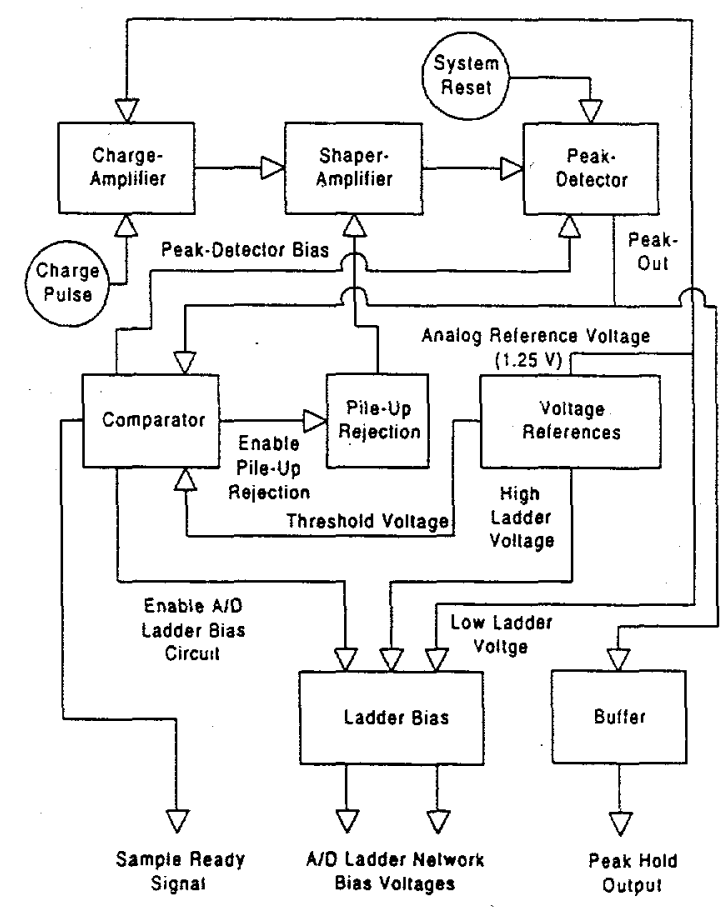

Figure 1. Block diagram of the puise processing ASIC. 


\section{DISCLAIMER}

This report was prepared as an account of work sponsored by an agency of the United States Government. Neither the United States Government nor any agency thereof, nor any of their employees, make any warranty, express or implied, or assumes any legal liability or responsibility for the accuracy, completeness, or usefulness of any information, apparatus, product, or process disclosed, or represents that its use would not infringe privately owned rights. Reference herein to any specific commercial product, process, or service by trade name, trademark, manufacturer, or otherwise does not necessarily constitute or imply its endorsement, recommendation, or favoring by the United States Government or any agency thereof. The views and opinions of authors expressed herein do not necessarily state or reflect those of the United States Government or any agency thereof. 


\section{DISCLAIMER}

Portions of this document may be illegible in electronic image products. Images are produced from the best available original document. 
The pulse processing ASIC is designed to work with a companion flash $\mathrm{ADC}$ and microcontroller to function as a complete ultra low-power gamma-ray pulse height spectrometer. In addition to its pulse processing functions, the ASIC also contains a circuit for biasing the external flash $A D C$ reference input. Finally, the ASIC contains a circuit to provide common and precise reference voltages to all other circuits in the ASIC.

The ASIC was designed to draw approximately $2 \mathrm{~mA}$ of current in the "idle mode" (no valid pulses present). When a pulse of appropriate amplitude triggers the circuit, its current draw will double. The circuit will then continue to draw 4 $\mathrm{mA}$ for the remainder of the pulse acquisition time $(<10 \mu \mathrm{s})$. Additional current will also be drawn during pulse acquisition to provide a reference for the external flash $A D C$. The amount of additional current drawn will depend on the ladder network used in the external ADC.

This design uses a band-gap voltage reference to set the operating dc voltages of the entire system. Since the band gap voltage is very stable over temperature and power supply voltage, the system can be designed to exploit this stability. The voltage references block generates three dc voltages for the system. The $1.25 \mathrm{~V}$ analog reference voltage $\left(\mathrm{V}_{\text {ref }}\right)$ sets the steady state dc voltages for the charge-amplifier output. Since the dc gain of the shaper-amplifier, peak-detector and buffer amplifier is unity, the charge-amplifier sets the dc operating voltages of these function blocks. Therefore the output of the buffer is a peak-held dc voltage relative to $V_{r e f}$ $(1.25 \mathrm{~V})$.

The voltage reference block provides a threshold voltage to the comparator. This voltage is slightly above $\mathrm{V}_{\text {ref }}$ and is adjusted to reject circuit noise. When the peak-detector output rises above the threshold voltage $\left(\mathrm{V}_{\text {urest }}\right)$ the pile-up rejection circuit and ladder bias circuit are enabled.

The voltage reference block also provides the high and low bias voltages to the $A D C$ resistor ladder bias network. The high voltage is adjusted to approximately $6.0 \mathrm{~V}$ while the low voltage is $V_{\text {ret }}(1.25 \mathrm{~V})$. The ladder bias block takes these low-current voltage references and applies these voltages to the low-value ladder resistor of the $\mathrm{ADC}$ converter. For the sake of minimizing system power consumption, the ladder bias circuit is enabled by the comparator when a nuclear pulse of $V_{\text {thrah }}$ is detected. The ladder resistor bias is maintained for the duration of the measurement which, in this design, is approximately $10 \mu \mathrm{s}$. After the sample has been digitized, the system is reset and returns to the lower power mode of operation (and remains in low power mode until another pulse of sufficient amplitude is produced by the detector).

The comparator block is used for four critical system functions: peak-detector bias control, ladder bias enabling, pile-up rejection and system noise rejection. These functions are enabled or disabled based on the sensing of a $V_{\text {thrah }}$ pulse input. The comparator provides bias to the output of the peak-detector such that the circuit is bi-directionally linear until the comparator senses an input pulse of amplitude greater than $\mathrm{V}_{\text {trrath }}$. When the comparator senses this input pulse, the peak-detector bias is removed and the peak- detector accurately follows the peak amplitude of the input. The peak-detector capacitor holds this voltage with very little change during the time interval required for $A D C$ conversion.

The comparator also enables the pile-up rejection circuit. The pile-up rejection circuit controls the ac gain of the shaper-amplifier. The shaper amplifier ac gain is allowed to be relatively large (20 to 30 ) when waiting for a $V_{\text {unros }}$ input pulse. After a pulse has tripped the comparator the shaperamplifier gain is allowed to stay high for a predetermined period of time (approximately $3 \mu$ s to $5 \mu \mathrm{s}$ ). This time is allowed to be long enough to completely capture one pulse. After this time, the ac gain of the shaper-amplifier is actively reduced to near unity. This gain reduction effectively eliminates the possibility of the desired pulse from being altered by a later arriving pulse greater than $V_{\text {tren }}$ in amplitude.

As stated earlier, the buffer is a unity gain amplifier. This amplifier is designed to drive relatively large capacitance loads of $20 \mathrm{pF}$. This is required because the flash $\mathrm{ADC}$ used in our system is essentially a string of paralleled gate inputs (vs. a single MOS input; which would be a relatively low input-capacitance). The base-currents of the input of the buffer stage were canceled by a current mirror arrangement. This technique minimizes the peakdetector droop caused by the base currents of the input stage. Base current cancellation typically results in very small effective input current although the sign of this current may be either positive or negative. Because it is nearly impossible to exactly cancel the input base currents, a very small net input current remains (though much smaller in amplitude than if one does not use a canceling technique at all).

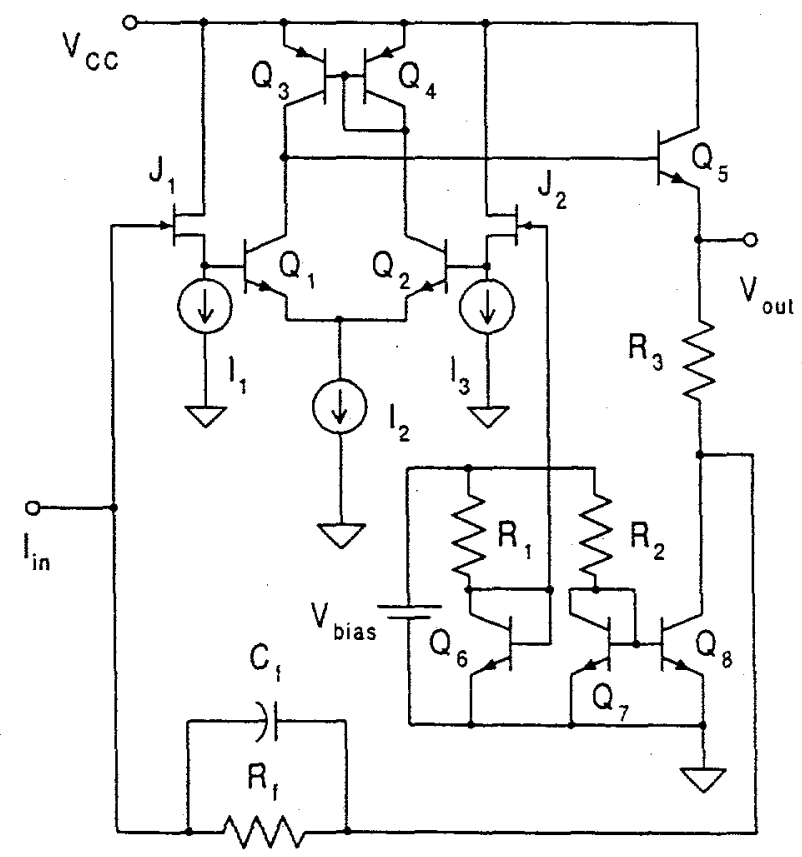

Figure 2. Schematic diagram of the charge sensitive amplifier section of the pulse processing ASIC. 


\section{CHARGE AMPLIFIER}

A simplified schematic for the charge amplifier in the pulse processing ASIC is shown in Figure 2. The design intention for this amplifier was to provide a large charge-gain to bias-current ratio and make the output dc voltage fixed to a band-gap reference voltage. This dc output subsequently becomes a reference voltage for the entire pulse-processing ASIC. The use of an active-load differential-amplifier provides a high-gain per unit bias current and allows for common mode noise rejection at the front-end of the amplifier. Current sources $I_{1}$ and $I_{3}$ use emitter resistors to minimize noise.

The external matched JFET pair $\left(J_{1}\right.$ and $\left.J_{2}\right)$ is used as a differential-amplifier configuration with $Q_{1}$ and $Q_{2}$ as the heart of the differential amplifier. $Q_{6}$ sets the bias voltage of the base of $Q_{2}$, and- thus by feedback- the gate of $J_{1}$. The current-mirror, $Q_{7}$ and $Q_{8}$ allow for $V_{\text {out }}$ to be biased by $V_{\text {bias }}$.

This amplifier was designed to operate at approximately $0.5 \mathrm{~mA}$ total current draw with $40 \mu \mathrm{A}\left(\mathrm{I}_{1}\right.$ and $\mathrm{I}_{3}$ ) of bias in each of the JFETS. This amplifier is an inverting transimpedance amplifier with $\mathrm{R}_{\mathrm{f}} / \mathrm{C}_{f}$ as the feedback impedance.

The differential-amplifier $\left(Q_{1}, Q_{2}, Q_{3}\right.$, and $\left.Q_{4}\right)$ is biased at $140 \mu \mathrm{A}\left(\mathrm{I}_{2}\right) . \mathrm{Q}_{s}$ is a buffer transistor that also supplies a required dc bias shift from the collector $Q_{1}$ and the output, $\mathrm{V}_{\text {out }}$. The amplifier output, $\mathrm{V}_{\text {ou }}$, is biased to the system $\mathrm{V}_{\text {ref }}$ $(1.25 \mathrm{~V})$ by the on-chip band-gap voltage reference. This bias voltage, represented by $V_{\text {bis }}$ sets the dc voltage at $V_{\text {our }}$. Transistors $\mathrm{Q}_{6}, \mathrm{Q}_{7}, \mathrm{Q}_{8}$ and resistors $\mathrm{R}_{1}, \mathrm{R}_{2}$, and $\mathrm{R}_{3}$ comprise a current-mirror bias circuit through which the dc voltage at $V_{\text {out }}$ is set. $V_{\text {our }}$ will be equal to $V_{\text {bis }}$ when $R_{1}, R_{2}$ and $R_{3}$ are equal values and $Q_{6}, Q_{7}$, and $Q_{8}$ are matched transistors. $R_{1}$, $R_{2}$ and $R_{3}$ were chosen to be $15 \mathrm{k} \Omega$. These resistor values provides a good compromise between circuit performance and power consumption. Ideally, for good circuit performance, $R_{3}$ should be small to allow the emitter of $Q_{5}$ to control the collector of $\mathrm{Q}_{\mathrm{s}}$ and the associated capacitance at this node, but this would require more power given this ASIC topology. The bias voltage is selected to be relatively low for good dynamic range yet large enough to provide adequate bias voltages around the amplifier. Given these constraints, $\mathrm{V}_{\text {bis }}$ was chosen to be $1.25 \mathrm{~V}$; a standard band-gap reference voltage. This amplifier is inherently very stable and of relatively high gain due to the single-stage of inverting-gain using high-impedance active loads. The amplifier circuit is phase-compensated by using a parasitic $1 \mathrm{pF}$ capacitor added to the base-collector capacitance of $Q_{1}$. The external JFETS are $\mathrm{n}$-channel epitaxial devices manufactured by Temic (part\# SST404) and have a very small $V_{\text {csions }}$ voltage of -2.5 $\mathrm{V}$ maximum and $-0.5 \mathrm{~V}$ minimum. The SST404 is a dual monolithic part in a surface mount package. The feedback resistor, $R_{r}$ is a $20 \mathrm{M} \Omega$ surface mount resistor. To maintain a large system charge-gain with minimum power consumption, the feedback capacitor $\mathrm{C}_{f}$ is the parasitic capacitance of $R_{r}$; this capacitance is approximately $0.15 \mathrm{pF}$.

\section{SHAPING AMPLIFIER}

A simplified block diagram of the shaper-amplifier design used in this ASIC is shown in Figure 3. The feedback components, $\mathrm{R} 1, \mathrm{Cl}, \mathrm{R} 2, \mathrm{C} 2$ and the impedance of $\mathrm{Q}$ c control theacgain of this circuit. The dc gain of this circuit is unity due to $C_{2}$ which is a large value $(0.1 \mu \mathrm{F})$ capacitor. $C_{1}$ is chosen in combination with $R_{1}$ and $R_{2}$ to provide the desired gain and filtering characteristics of the shaper-amplifier function. $\mathrm{V}_{\text {gin }}$ is a digital "like" signal provided by the comparator and pile-up rejection block; $\mathrm{V}_{\text {gin }}$ either holds $Q_{1}$ in saturation or cutoff. The transistor impedance is very low when the device is in saturation $\left(\mathrm{V}_{\text {gun }}\right.$ high) and therefore theacgain of the amplifier is set by the passive feedback components. When $\mathrm{V}_{\text {zan }}$ is low, the transistor is off and the collector to emitter impedance is very high forcing theacgain of the amplifier to unity. This gain control allows the rejection of charge- pulses after the initial sampling of a desired pulse. A gain ratio of approximately 20:1 to $40: 1$ is easily obtained using this technique. This range is typically enough to reject undesired pulses during the processing of a captured sample. In this design $R_{1}$ was chosen to be $30 \mathrm{k} \Omega$ with $C_{1}$ equal to approximately $10 \mathrm{pF}$. $\mathrm{R}_{2}$ is set to $1.5 \mathrm{k} \Omega$ to provide the desired overall charge-gain of approximately $110 \mathrm{mV} / \mathrm{fC}$ and allow for a large,relatively high to low gain ratio.

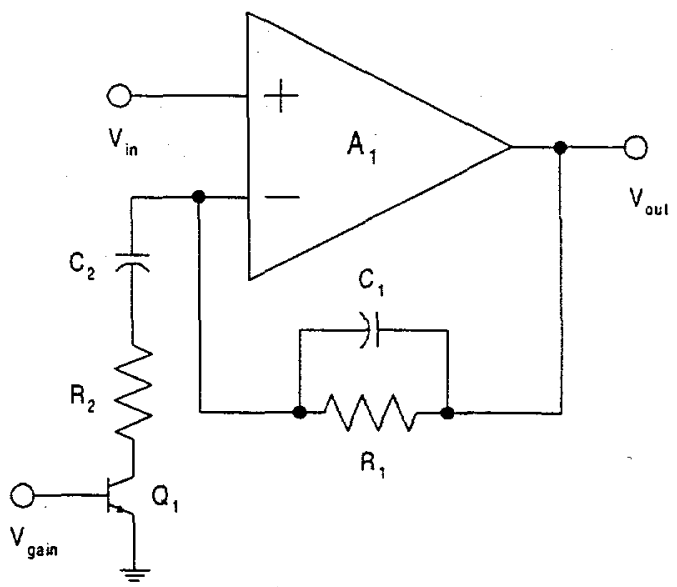

Figure 3. Schematic diagram of the shaping amplifier section of the pulse processing ASIC.

The analog approach to pile-up rejection just described has one serious flaw. When $Q_{1}$ is turned off (immediately after a charge pulse has been peak-held) a small parasitic voltage pulse is generated at the output of the shaperamplifier, effectively limiting the low-level sensitivity of this design. For this ASIC, the low-level limit due to this parasitic pulse is approximately $3 \mathrm{fC}$. Since this system uses a flash $A D C$, the system acquires a sample and resets in less than $15 \mu \mathrm{s}$. Thus, it is possible to use the ASIC without the pile-up rejection circuit if the rate of input pulses is relatively low. A redesign of the pile-up rejection block is being considered to eliminate the parasitic pulse. This new design leaves the shaper-amplifier gain constant but disables the peak-detector circuit instead. This new design appears to 
have no parasitic glitches and may be a better solution than the one presented here.

\section{ADC REFERENCE INPUT BIASING}

The pulse processing ASIC is designed to work with another circuit module which incorporates an $A D C$ and histogramming memory features. The ADC in the companion module is an 8 bit flash $\mathrm{ADC}$ which utilizes a rather low impedance resistor "ladder" network on its reference inputs. Thus biasing the ladder consumes a sizable amount of power. To minimize system power consumption, the ASIC described here incorporates a ladder bias supply which biases the ADC only when the comparator has detected a pulse of valid amplitude.

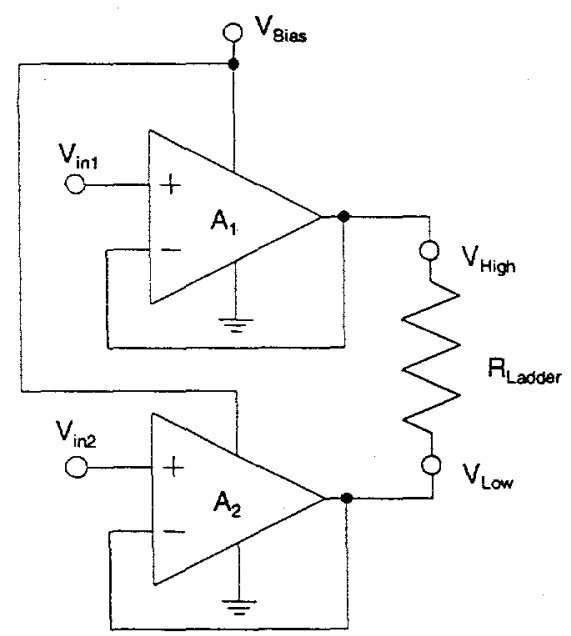

Figure 4. Diagram of the circuit used to provide biasing to the reference input of an external flash ADC.

A diagram of the circuit used in the ASIC to supply the required $\mathrm{dc}$ reference voltages for the flash $A D C$ ladder resistor network is shown in Figure 4.

This circuit comprises two op-amp circuits, $A_{1}$ and $A_{2}$ performing a dual follower function. $A_{1}$ applies $V_{\text {High }}$ to the top of $R_{\text {ladder }}$ while $A_{2}$ applies $V_{\text {Low }}$ to the bottom of $R_{\text {Ladder }}$. These voltages represent the highest and lowest measurable voltages the $A D C$ will process at its input. Since the ladder resistor can range from $2 \mathrm{k} \Omega$ to $5 \mathrm{k} \Omega$ and the voltage range of the input will be approximately 5 volts, $2.5 \mathrm{~mA}$ (max) is required to bias this resistance. To minimize the system power requirements it is highly desirable to apply this bias voltage for the least amount of time possible. Thus this circuit is designed to apply $V_{\text {High }}$, and $V_{\text {Low }}$ to $R_{\text {tedder }}$ only when a pulse has triggered the comparator and to be reset to zero bias shortly after the acquisition of the pulse. This is accomplished by using the comparator to bias the circuit via the $\mathrm{V}_{\text {tas }}$ node when a pulse trips the threshold voltage.

When biased "on", the opamps require approximately $160 \mu \mathrm{A}$ of current each. They are designed to turn on quickly, have low-offset voltages and operate at minimum possible current.

\section{PEAK DETECTOR}

The final stage of analog pulse processing that is performed by the ASIC is the peak sample and hold function. In conventional pulse processing systems the peak sample and hold is usually incorporated as part of the ADC, but we chose to incorporate as part of the pulse processing ASIC to minimize power consumption.

A simplified schematic of the peak detector used in this ASIC design is shown in Figure 5.

$I_{1}$ is approximately $120 \mu \mathrm{A}$ and $L_{2}$ is approximately 50 $\mu \mathrm{A}$. $\mathrm{I}_{\mathrm{idk}}$ is $5 \mu \mathrm{A}$ or $0 \mu \mathrm{A}$ and is controlled by the comparator. $\mathrm{I}_{\text {idec }}$ is turned off when the output of the comparator senses an $\mathrm{V}_{\text {unrash }}$ input pulse. $\mathrm{C}_{\text {pear }}$ is an external $400 \mathrm{pF}$ capacitor. A pnp $\left(Q_{1}, Q_{2}\right)$ based differential-amplifier was chosen for this design. The input is biased at the system $V_{r e f}(1.25 \mathrm{~V})$ from the shaper-amplifier. The input is on the base of $Q_{1}$ and the feedback from the peak hold capacitor, $\mathrm{C}_{\text {pak }}$, is feedback to the base of $Q_{2}$. After a positive going peak has been held on $\mathrm{C}_{\text {peak }}$ and the input returns to base line dc, $\mathrm{Q}_{2}$ will be cutoff and therefore the base current of $Q_{2}$ will be near zero and not discharge $\mathrm{C}_{\text {paak. }}$. Using this topology with an npn design would leave an active base on $\mathrm{C}_{\text {pak }}$ and allow a very significant discharge path. $Q_{3}$ is an emitter follower stage that guarantees that $Q_{2}$ cannot be saturated. When the circuit is idling or following a positive going signal $Q_{3}$ biases the collector to emitter voltage of $\mathrm{Q}_{2}$ at approximately one diode drop. With $I_{\text {idte }}$ "on" this circuit is essentially a low-offset, unidirectional

follower circuit. For good low-level accuracy and low overshoot $I_{\text {idte }}$ is sourced from emitter of $Q_{5}$ until the threshold comparator trips and then $\mathrm{I}_{\text {idte }}$ is reduced to zero and a peakhold is obtained. The offset of this design is also low because the $Q_{3}$ base-current is relatively small compared to the collector currents of $Q_{1}-Q_{2}$; allowing the differential amplifier to control the peak very accurately. The overshoot is essentially zero because the circuit is actively servoing the $\mathrm{C}_{\text {pak }}$ with all devices on and in the linear region of operation before $V_{\text {thresh }}$ is sensed. When $I_{\text {idle }}$ is turned off, the emitter current of $Q_{5}$ is determined by the charge current into $C_{\text {park }}$ and the circuit is very linear in operation. When the peak of the input has occurred and the input is decreasing, $Q_{2}$ and $Q_{5}$ are turned off and the current-leakage paths from $C_{\text {palk }}$ are very low. 


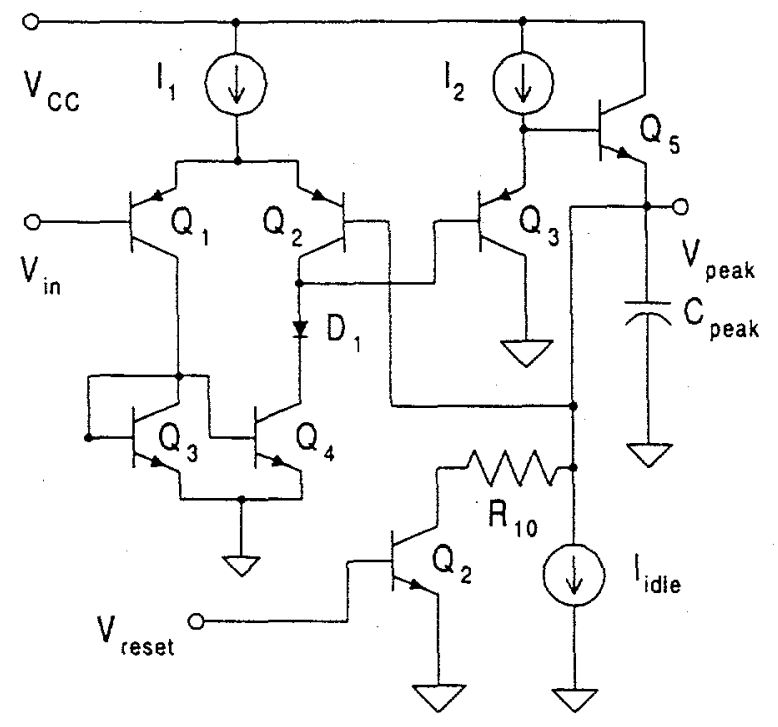

Figure 5. Schematic diagram of the peak sample and hold circuit used in the pulse processing ASIC.

One problem with this type of peak-detector design is that there is a limit to the amplitude of the detected signal. This limitation is the reverse breakdown voltage of $\mathrm{Q}$, (approximately $5.6 \mathrm{~V}$ ). To make this limitation have the least impact on the system performance, diode $D_{1}$ was added. This arrangement holds the collector voltage, $Q_{2}$, at one diode drop above ground plus the saturation voltage of $\mathrm{Q}_{4}$ after a peak has been held and $Q_{4}$ is on hard. The addition of $D_{1}$ allows one diode drop greater peak voltage out before reverse breakdown of $Q_{5}$ occurs than if $D_{1}$ was not used (approximately 6.9 volts compared to 6.3 volts).

Finally, $Q_{2}$ and $R_{10}$ make up a reset network. The system reset circuitry biases $V_{\text {rese }}$ high to remove the charge on $C_{\text {peak }}$ while $R_{10}$ limits the current $Q_{5}$ can provide to ground during the reset process.

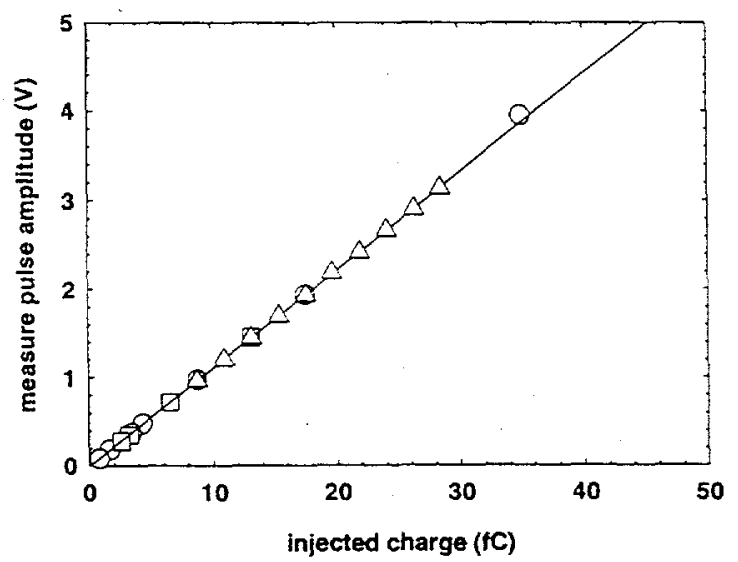

Figure 6. Measured output of the ASIC amplifier (charge and shaper) versus known charge injected to input.

\section{RESULTS}

A version of the pulse processing circuit was built using Honeywell's ALB1A bipolar ASIC fabrication process. The resulting ASIC dice were $4 \mathrm{~mm} \times 5 \mathrm{~mm}$ in size and were packaged in 40 pin leadless chip carriers (LCCs) for initial testing. The packaged ASICs were attached to a standard FR4 printed circuit board with surface mount external components for testing. Radiation detectors were attached to the ASIC test board using microdot and BNC connectors.

The gain and linearity of the ASIC module were measured by injecting a voltage pulse from an Ortec 419 Precision Pulse Generator into the input of the ASIC using an Ortec "charge terminator" ( $2 \mathrm{pF}$ capacitor terminated $100 \Omega$ to ground). The tail pulse generator and terminator had previously been calibrated with a silicon detector and the amount of charge injected into the ASIC was known to good precision. After injecting the fixed amplitude charge pulses into the ASIC input, the distribution of pulses produced at the output of the shaping amplifier was monitored with an Aptec Series 5000 multi-channel analyzer (MCA). The centroid of the peaks produced in the pulse height spectrum were used to determine the gain and linearity of the ASIC (Figure 6).

The output of the shaper amplifier was plotted versus a calibrated fixed charge pulse provided to the preamplifier input. Open symbols are measured laboratory data and the solid line is a linear fit to the data using a "least squares" algorithm. These data allow a determination of the overall system (preamplifier and shaping amplifier) gain and linearity. - The slope of the least squares fit yields a system gain of $110 \mathrm{mV} / \mathrm{fC}$ ( $18 \mu \mathrm{V} / \mathrm{electron}$ ), close to the original design specification. The correlation coefficient of the fit indicates a linearity of better than $0.1 \%$.

By measuring the width of the peaks in the pulse height spectra (using the apparatus just described), the noise of the circuit (ENC) was found to be 538 electrons rms (1270 electrons FWHM); well within the design specifications.

The ASIC was also operated in its intended mode- as a gamma-ray spectrometer- using two different detectors. The pulse height spectrum shown in Figure 7 was obtained by irradiating a silicon $\mathrm{p}-\mathrm{i}-\mathrm{n}$ photodiode (Hamamatsu S1223) with a ${ }^{57} \mathrm{Co}$ source. The pulse processing ASIC was used to amplify and shape the pulses from the detector.

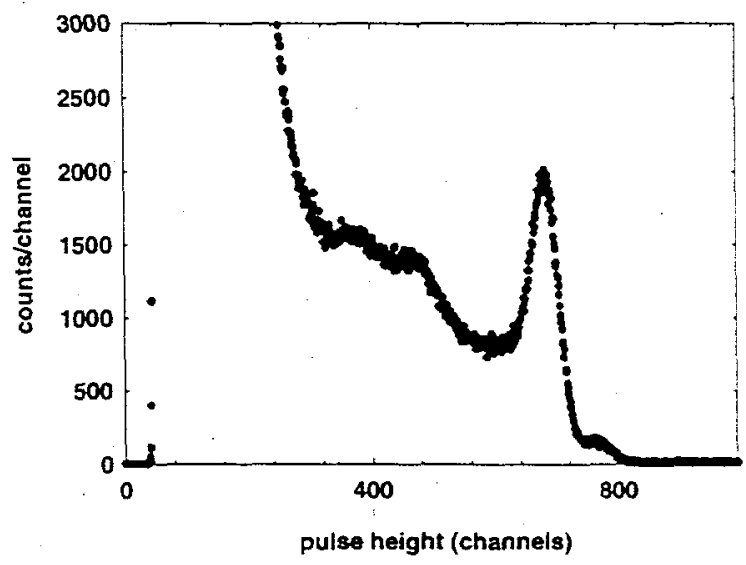

Figure 7. Pulse height spectrum of a ${ }^{57} \mathrm{Co}$ source taken with a silicon $\mathrm{p}-\mathrm{i}-\mathrm{n}$ detector and the electronics described in the text. 


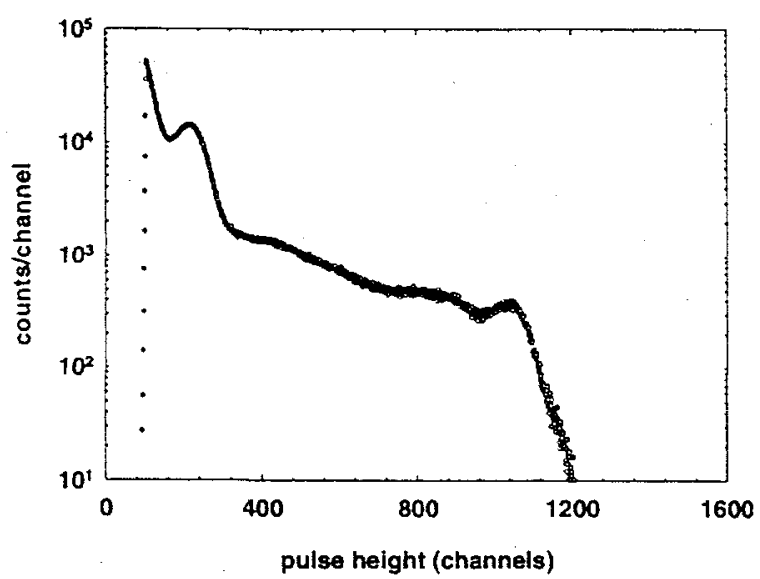

Figure 8. Pulse height spectrum of ${ }^{133} \mathrm{Ba}$ taken with a CZT detector and the electronics described in the text.

The same commercial pulse height analyzer (Aptec Series 5000) was used to histogram the pulse amplitudes and a ${ }^{57}$ Co source was used to irradiate the detector. Figure 8 shows a pulse height spectrum obtained with a CZT detector attached to the detector, and irradiated with a ${ }^{133} \mathrm{Ba}$ source.

The pulse height spectrum in Figure 8 was obtained by irradiating a $15 \mathrm{~mm} \times 15 \mathrm{~mm} \times 2 \mathrm{~mm}$ CZT detector with photons from ${ }^{133} \mathrm{Ba}$ and reading out the detector with the ASIC described in the text. The CZT detector was of "spectroscopic grade" and was operated at a bias of $100 \mathrm{~V}$.

The peak-detector circuit was also evaluated for droop (decay of the peak held voltage as a function of time), offset and overshoot. The droop measures approximately 100 $\mu \mathrm{V} / \mu$ s and the offset is less than $10 \mathrm{mV}$, this offset includes the buffer circuit. The peak-detector exhibits no measurable overshoot. The droop is caused by the comparator circuit input bias current. This droop is perfectly acceptable for our application due to the rapid digititization of the flash $A / D$. The peak detector need only hold the sample for approximately $10 \mu \mathrm{s}$ before digitization, which implies a $1 \mathrm{mV}$ maximum error; this is well below the overall system noise floor.

\section{SUMMARY}

An ultra-low power consumption pulse processing circuit has been designed, constructed, and tested. The circuit is designed for use in an unattended monitoring application where multi-year operation from batteries is essential.

An ASIC embodiment of the circuit was constructed using Honeywell's ALB IA bipolar ASIC fabrication process. The resulting ASICs were tested and found to meet all of the design criteria necessary for use in the unattended monitoring application.

\section{ACKNOWLEDGMENTS}

We gratefully acknowledge support for this work from Sandia National Laboratories' Integrated Nuclear Materials Monitoring Program (INuMM).

Sandia is a multi-program laboratory operated by Sandia Corporation, a Lockheed Martin Company, for the U.S. Department of Energy under contract number: DE-AC0494AL 85000 .

\section{REFERENCES}

[1] V. Radeka, P. Rehak, S. Rescia, E. Gatti, A. Longoni, M. Sampietro, P. Holl, L. Strüder, and J. Kemmer, Design of a Charge Sensitive Preamplifier on High Resistivity Silicon, IEEE Trans. Nuc. Sci., NS-35, p. 155, (1988).

[2] F. Evangelisti, G. Orsi, G. Venture, A Low Noise, Very Low Power Charge Sensitive Amplifier for Space Applications, Rev. Sci. Inst., 44, p. 738 (1973)

[3] G.F. Knoll, Radiation Detection and Measurement, 2nd ed., Wiley, New York, (1989).

[4] N. Tsoulfanidis, Measurement and Detection of Radiation, Hemisphere Pub., New York, (1983).

[5] V. Radeka, Low-Noise Techniques in Detectors, Ann. Rev. Nucl. Part. Sci., 38, p. 217, (1988). 